Philosophie ANTIQUE

\section{Philosophie antique}

Problèmes, Renaissances, Usages

$10 \mid 2010$

Philosophie et mathématiques

\title{
«Ceux qui acceptent des Idées de toutes choses »
}

Sur l'interprétation de Parménide 130b3-e4 dans l'Antiquité tardive

\section{Pieter d'Hoine}

\section{(2) OpenEdition}

Journals

Édition électronique

URL : https://journals.openedition.org/philosant/2328

DOI : $10.4000 /$ philosant.2328

ISSN : 2648-2789

Éditeur

Éditions Vrin

\section{Édition imprimée}

Date de publication : 30 octobre 2010

Pagination : 227-254

ISBN : 978-2-7574-0179-8

ISSN : $1634-4561$

Référence électronique

Pieter d'Hoine, « "Ceux qui acceptent des Idées de toutes choses » », Philosophie antique [En ligne],

10 | 2010, mis en ligne le 11 juillet 2019, consulté le 02 décembre 2022. URL : http://

journals.openedition.org/philosant/2328; DOI : https://doi.org/10.4000/philosant.2328

\section{(c) (i) (9)}

Creative Commons - Attribution - Pas d'Utilisation Commerciale - Pas de Modification 4.0 International - CC BY-NC-ND 4.0

https://creativecommons.org/licenses/by-nc-nd/4.0/ 


\title{
«CEUX QUI ACCEPTENT DES IDÉES DE TOUTES CHOSES "
}

\author{
Sur l'interprétation de Parménide 130b3-e4 dans l'Antiquité tardive \\ Pieter D'HOINE* \\ K.U. Leuven
}

RÉSUMÉ. Chez les commentateurs platoniciens de l'époque impériale, l'un des problèmes majeurs liés à la théorie des Idées concernait le domaine d'application de cette doctrine. L'exégèse de la première partie du Parménide de Platon donnait occasion à diverses discussions sur ce sujet. Le Commentaire de Proclus sur le Parménide est sans doute la plus précieuse source qui soit parvenue de l'Antiquité jusqu'à nous pour la reconstitution de ces débats. Alors que la grande majorité des commentateurs anciens étaient convaincus que les Idées ont un domaine assez restreint, le Commentaire de Proclus nous révèle l'existence d'une lecture alternative, selon laquelle il y a des Idées de «toutes choses ». Proclus relie cette interprétation alternative en particulier à l'existence des Idées de choses mauvaises et d'individus, probablement parce que ces deux classes d'objets étaient vraiment les dernières choses dont un platonicien orthodoxe voudrait admettre des Idées. Amélius est le seul néoplatonicien connu pour avoir soutenu l'existence des Idées de choses mauvaises. Dans cet article j'essaie de montrer que, selon toute vraisemblance, Amélius est également la cible de la réfutation des Idées d'individus par Proclus dans son Commentaire. La conclusion qui s'ensuit naturellement est qu'Amélius était très probablement l'un de ces philosophes qui, d'après Proclus, admettaient des Idées « de toutes choses ».

Summary. Among the Platonic commentators of the imperial age, one of the key problems connected to the theory of Forms concerned the range of application of this doctrine. The exegesis of the first part of Plato's Parmenides provided the occasion for various discussions of this topic. Proclus' Commentary on the Parmenides is no doubt the most precious source that has come down to us from Antiquity for the reconstruction of these debates. While the vast majority of ancient commentators seem to have agreed that there cannot be Forms of all things

* Chargé de recherche du Fonds de la Recherche Scientifique-Flandre (FWO). Je tiens à remercier vivement Caroline Macé de m'avoir assisté pourla version française de ce texte.

Philosophie antique, $\mathrm{n}^{\circ} 10$ (2010), 227-254 
sensible, Proclus' commentary teaches us that an alternative reading, according to which the theory of Forms is to be regarded all-extensive, has also been proposed. Proclus connects the doctrine that there are Forms 'of all things' especially with Forms of evils and individuals, presumably because these two classes of items were really the last things of which an orthodox Platonist would want to accept Forms. Amelius is the only Neoplatonist known to have defended the existence of Forms of evils. In this paper I try to show that, in all likelihood, Amelius is also the target of Proclus' refutation of Forms of individuals in the Commentary. These considerations point to the conclusion that very likely. Amelius was one of those philosophers who in Proclus' view accepted Forms 'of all things'. 
Dans la première partie du Parménide de Platon, la théorie des Idées que nous connaissons d'après les dialogues de la maturité fait l'objet d'une analyse critique de la part de Parménide et du jeune Socrate. Le premier problème discuté concerne le domaine d'application des Idées. En effet, une fois admise l'existence d'Idées séparées des choses sensibles, il convient de se demander quelles choses, si ce n'est pas le cas de toutes, participent à de telles Idées. En Parménide, 130b3-e4, le philosophe d'Élée demande tout d'abord si Socrate accepte des Idées de relations et propriétés générales, telles que la Ressemblance, l'Unité, la Pluralité et «toutes ces choses qu'[il] vient d'entendre de Zénon 》 $(\pi \alpha \dot{\alpha} \nu \tau \alpha$

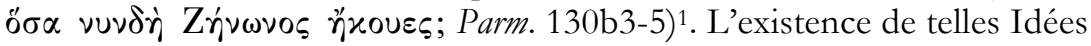
est pour Socrate hors de doute. Ensuite, Parménide évoque les Idées de la Justice, de la Beauté, du Bien « et d'autres choses de ce genre » (Parm. 130b7-9). Socrate les accepte également sans hésitation. Seule la troisième question de Parménide suscite quelque réserve chez Socrate : existe-t-il une Idée séparée de l'Homme, du Feu et de l'Eau (Parm. 130c1-2) ? Le jeune philosophe confesse être quelque peu décontenancé par ces exemples d'espèces et d'éléments naturels : il n'est pas totalement convaincu qu'il faille admettre des Idées dans ce cas (Parm. 130c3-4). Finalement, Parménide invite Socrate à considérer une dernière classe d'objets. Il souhaite savoir si Socrate accepte des Idées des objets les plus vils et les plus triviaux, comme le poil, la boue et la saleté (Parm. 130c5d2). La réponse de Socrate laisse quelque peu perplexe : après un rejet catégorique de telles Idées, le jeune philosophe admet cependant que la question l'a troublé plus d'une fois. En effet, si peu plausible que puisse être l'existence d'Idées de ces choses sans valeur, il est peut-être

1. Comme l'attestent les passages Parm. 127c2 sqq. et 136a4-b6, les choses mentionnées par Zénon doivent être des notions générales comme la ressemblance et la dissemblance, l'unité et la pluralité, le repos et le mouvement, etc. 
préférable qu'il n'y ait qu'un seul et unique principe qui permette d'expliquer toute la réalité. Plutôt que de se torturer l'esprit avec de telles questions, Socrate préfère toutefois limiter sa recherche aux Idées dont il est sûr, à savoir les deux premières classes d'Idées qui ont été discutées : les termes généraux désignant des relations et les notions éthiques ou esthétiques. Ces deux classes constitueront en effet l'objet principal des recherches menées à propos des Idées dans la première partie du Parménide. C'est à Parménide cependant qu'il revient d'avoir le dernier mot sur ce sujet. Sans étendre explicitement le domaine des Idées au-delà des limites établies, il reproche à Socrate de mêler indûment la question sur l'extension du domaine des Idées à des considérations de valeur. En cela, Parménide considère que Socrate s'est laissé abuser par les opinions de la foule (Parm.130e1-4).

Le passage que je viens de présenter est d'un grand intérêt pour la «théorie » platonicienne des Idées et son histoire dans l'antiquité - non seulement parce qu'il s'agit du seul passage chez Platon où le domaine des Idées est examiné explicitement, mais aussi à cause des débats que ce texte a suscités dans la tradition platonicienne postérieure. Ce passage apporte, il est vrai, plus de questions que de réponses. Ni le jeune Socrate ni le vieux Parménide ne proposent de réponse explicite aux difficultés soulevées. Et même s'ils l'avaient fait, nous pourrions nous demander lequel des deux interlocuteurs ou même si l'un des deux représente l'opinion de Platon en la matière. Ces circonstances ont poussé Franco Ferrari à comprendre le passage comme une confrontation entre l'eticismo de Socrate et l'ontologismo de Parménide ${ }^{2}$. En effet, les Idées vis-à-vis desquelles Socrate montre ici le plus de certitude appartiennent au domaine dans lequel le Socrate historique avait une grande expertise : les termes comportant un jugement de valeur de caractère éthique ou esthétique et les relations générales. Ce sont aussi les exemples d’Idées privilégiés par Socrate dans les dialogues de jeunesse et de maturité3. En outre, il est bien connu que le Socrate historique avait peu d'intérêt pour

2. Ferrari 2004, p. 52-56. Pour d'autres vues sur ce passage, voir Cornford 1939, p. 81-84 ; Miller 1986, p. 44-47 ; Brisson, 1994, p. 34-35 ; Gill 1996, p. $23-24$; Allen 1997, p. 119-124; Fronterotta 2001, p. 118-124.

3. Sur les Idées de relations, voir p. ex. Phaed. 74a3 sqq. et 100b6; Resp. V, 479b3-7 et VII, 524d9 sqq. Cf. Theaet. 186a6-7 et Soph. 254b8 sqq. Les notions de valeur font l'objet de discussions dans la plupart des dialogues socratiques : p. ex. la beauté dans Hipp. Maj., la piété dans Euthyphr., la vertu dans Men. et Prot., la sagesse dans Charm. et la justice dans Resp. Des Idées de telles notions sont postulées p. ex. dans Phaed. 65d4-7, 100b5-6; Theaet. 186a9 ; Symp. 210e2 ; Hipp. Maj. 286c8-d2 ; Phaedr. 247d5-e4 ; Resp. V, 476a1-8, 478e7-479a7 ; cf. Resp. VI, 504e7-505b3 ; Phil. 15a5-6 ; Epist. VII, 342d4-5. 
les questions de philosophie naturelle 4 . Ceci peut expliquer pourquoi les Idées de substances naturelles apparaissent seulement dans les dialogues tardifs 5 et pourquoi Socrate dans le Parménide hésite à accepter de telles Idées. Quant aux Idées d'objets vils ou triviaux, aucun exemple ne peut en être trouvé dans les dialogues. Somme toute, il n'est pas déraisonnable de supposer que tant les affirmations que les déroutantes hésitations du jeune Socrate sont dues à la volonté de Platon de donner à cette dramatis persona une sorte d'épaisseur historique - même si présenter Socrate ou Parménide comme acceptant des Idées tout simplement n'en demeure peut-être pas moins une fiction historique ${ }^{6}$.

Cette position de Socrate est mise en contraste, dans le dialogue, avec l'approche plus ontologique de Parménide : s'il y a une réalité intelligible séparée du monde que nous voyons, alors pourquoi ne pas postuler une Idée pour chaque groupe de choses auxquelles nous appliquons le même nom $^{7}$ ? Dès lors, des Idées d'espèces et d'éléments naturels sont tout aussi acceptables que des Idées de termes relatifs. En outre, d'un point de vue ontologique, des considérations de valeur - telles que celles que Socrate oppose aux Idées d'objets triviaux - ne sont pas recevables. C'est peut-être la raison pour laquelle, dans les dialogues tardifs de Platon, les Idées de substances naturelles sont bien présentes et qu'il est fait au moins deux fois allusion aux Idées d'objets triviaux, bien qu'elles ne soient pas explicitement reconnues ${ }^{8}$. Il n'est donc pas invraisemblable que, comme la plupart des interprètes modernes le pensent, le Parménide du dialogue tende à reculer les frontières du monde intelligible pour qu'il puisse inclure toutes choses. Ce que le dialogue nous livre, ce sont, dès lors, deux visions contrastées sur l'étendue du « monde des Idées » : celle de Socrate, d'une part, pour qui les Idées demeurent étroitement liées à des questions d'épistémologie et de morale; et celle de Parménide,



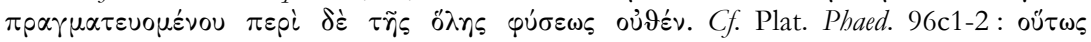


d1.

5. Cf. Tim. 51b2-c5, 30c-31b (le క̄̃ov intelligible, qui comprend tous les êtres vivants) ; Phil. 15a4-5 ; Epist. VII, 342d5-7. Pourtant, déjà dans le Ménon (72a8-b7) il y a

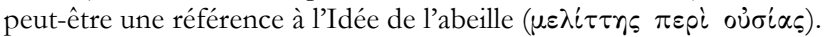

6. Cf. Arist. Metaph. A, 9, 987b1-10 ; $\Lambda, 4,1078 \mathrm{~b} 17-31$. Je ne crois pas que Coxon 1999, p. 7-25, ait raison d'insister sur la fiabilité du Parménide par rapport à la figure historique de Socrate.

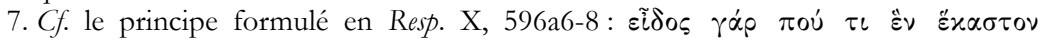

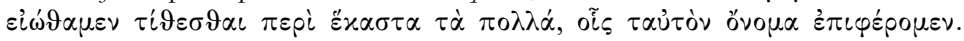

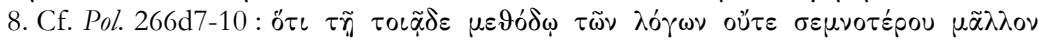

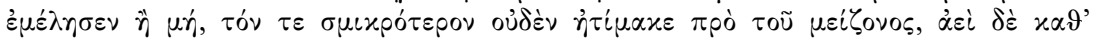




d'autre part, qui est prêt à pousser l'hypothèse des Idées jusqu'à ses conséquences ontologiques les plus radicales et, apparemment, les plus absurdes.

\section{Parménide 130b3-e4 dans l'Antiquité tardive}

Le passage présenté ici est le locus classicus pour toutes les discussions, tant anciennes que modernes, sur le domaine de la théorie platonicienne des Idées. Le problème a reçu beaucoup d'attention de la part des platoniciens de l'antiquité tardive, dont le but était de formuler les remarques éparses de Platon sur les Idées en une doctrine cohérente. Notre source la plus précieuse à cet égard est le Commentaire sur le Parménide écrit au $\mathrm{V}^{\mathrm{e}}$ siècle de notre ère par le néoplatonicien Proclus, le seul commentaire antique subsistant sur la première partie du dialogue. Dans le quatrième livre de son commentaire, Proclus discute Parménide 131a4-7, où Parménide commence à traiter des problèmes de la participation. Dans ce contexte le commentateur néoplatonicien revient sur la question de savoir comment les commentateurs précédents du dialogue ont interprété la discussion sur le domaine des Idées :

Le premier argument est construit par eux [scil. les interprètes précédents] à peu près comme ceci : s'il y a des Idées, il y a des Idées de toutes choses; or il n'y en a pas de toutes choses, comme tu [Socrate] le reconnais toi-même ; il n'y a donc pas d'Idées. Ceux qui admettent qu'il y a des Idées de toutes choses acceptent la proposition hypothétique

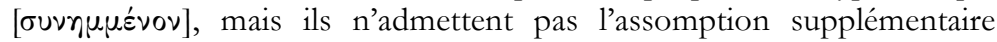
$[\pi \rho o ́ \sigma \lambda \eta \psi(\nu]$, et disent que pour cette raison Socrate est justement critiqué, parce qu'il ne pose pas d'Idées de toutes choses. Ils approuvent Parménide et considèrent que les réserves de Socrate sont infondées. D'autres, à l'inverse, acceptent l'assomption supplémentaire, mais repoussent comme non correcte la proposition hypothétique: car ils disent qu'il est faux de dire que "s'il y a des Idées, il y a des Idées de toutes choses ", mais que l'assomption "or il n'y a pas des Idées de toutes choses » est vraie ; ceux-là approuvent Socrate dans son refus, et repoussent la critique de Parménide?

Proclus distingue deux interprétations de l'argument du Parménide. Ces deux interprétations, cependant, se basent sur la même construction

9. In Parm. IV , 856.13-25 ; cf. le passage parallèle en In Parm. IV, 837.15-22. Toutes les références à l'In Parm. de Proclus renvoient à la nouvelle édition de Steel 2007-2009. Pour les traductions de ce commentaire, j'ai fait usage de la traduction de Chaignet 1900-1903, que je corrige ou modifie à de nombreux endroits. 
de l'argument. En fait, les deux groupes d'exégètes réduisent l'argument au syllogisme hypothétique suivant (modus tollens) :

H. Si les Idées existent, il y a des Idées de toutes choses m. Il n'y a pas d'Idées de toutes choses

C. Les Idées n'existent pas

Selon les interprètes auxquels Proclus se réfère, les deux prémisses de l'argument correspondent aux positions respectives des deux interlocuteurs du dialogue. C'est-à-dire que le doute de Socrate à propos des Idées des espèces naturelles et son refus des Idées de trivialités doivent être pris comme une défense, de la part de Socrate, d'une version restreinte de la théorie des Idées $(=\mathrm{m})$. En revanche, le reproche final adressé par Parménide à Socrate peut être interprété comme une objection ad hominem contre l'opinion de ce dernier. L'affirmation qu'il y a des Idées séparées des choses visibles - comme Socrate le croit - exige donc, selon le Parménide du dialogue, qu'il existe des Idées de toutes les choses visibles $(=\mathrm{H})$.

De toute évidence aucun vrai platonicien ne pourrait souscrire à la conclusion de l'argument, car cela conduirait à la négation de l'une des doctrines absolument centrales du platonisme dogmatique ancien: la théorie des Idées. Toujours à l'époque de Proclus, les principaux opposants à la théorie des Idées étaient les péripatéticiens ${ }^{10}$. Ce n'est donc pas nécessairement un hasard si la ligne de pensée attribuée à Parménide dans le texte ci-dessus a la même teneur qu'une objection aristotélicienne bien connue contre la théorie des Idées transcendantes dans la Métaphysique et dans le De Ideis ${ }^{11}$. Selon le Stagirite, l'un des problèmes liés aux arguments académiques pour démontrer l'existence des Idées est que - si du moins ces arguments sont valides - ils prouvent trop, en ce sens qu'ils introduisent des Idées même de choses pour lesquelles les platoniciens n'admettaient pas d'Idées. Par exemple, les soidisant «arguments tirés des sciences » amènent à postuler des Idées des artefacts, l'argument « de l'unité d'une multiplicité » conduit quant à lui à

10. Cela est confirmé par le fait que les arguments de Proclus en faveur de l'existence des Idées sont clairement dirigés contre Aristote. Voir p. ex. d'Hoine 2008. Voir aussi p. ex. In Parm. IV, 972.26-973.9.

11. Arist. Metaph. A, 9, 990b8-17 (cf. $\Lambda, 4,1079 \mathrm{a} 4-13$; De Id. 79.3-85.13). Que ces arguments étaient toujours connus (probablement à travers Alexandre d'Aphrodise) et discutés à l'époque de Proclus est confirmé par la réfutation que Syrianus en fait dans son Commentaire sur la Métaphysique (p. ex. In Metaph. 109.28-112.6). Pour ce qui concerne Proclus, voir p. ex. sa critique de l'argument «de l'objet de pensée » en In Parm. II, 731.18-22. 
des Idées des négations, celui "de l'objet de pensée » à des Idées des individus, etc. Par conséquent, Aristote peut disqualifier la théorie en raison de son inconsistance apparente, vu que les platoniciens, d'un côté, croient à l'existence d'Idées telles que celles qui sont introduites par ces arguments, alors même que, d'un autre côté, ils restreignent le domaine intelligible de manière à exclure les Idées des individus, des négations et des $\operatorname{artefacts}^{12}$. Si ces platoniciens acceptent les Idées, ils devraient les accepter dans tous les cas où leurs arguments s'appliquent - ce qu'ils ne font pas. Malgré le caractère plus particulier de ces arguments, la conclusion implicite du Stagirite est la même que celle attribuée à Parménide par les anciens interprètes du dialogue homonyme : les Idées n'existent pas. On peut donc soupçonner que ces exégètes de Platon ont développé cette interprétation afin de renforcer leur opinion que, dans la première partie du Parménide, Platon avait déjà anticipé et réfuté toutes les objections postérieures contre sa propre théorie des Idées ${ }^{13}$. Cela exige, cependant, que l'argument du Parménide, qui présente une réfutation des Idées, puisse lui-même être réfuté. Or, afin de refuser la conclusion d'un syllogisme valide, il est nécessaire d'attaquer au moins l'une de ses prémisses. Dans le texte cité plus haut, Proclus décrit les deux stratégies qui ont été adoptées pour ce faire par ses prédécesseurs. La première stratégie consiste à accepter la proposition hypothétique $(\mathrm{H})$ de l'argument tout en réfutant la prémisse mineure $(\mathrm{m})$. Cette interprétation assume la position de Parménide dans la discussion et mène donc à une application universelle de la théorie des Idées. La seconde stratégie fait le contraire : tout en réfutant la proposition hypothétique $(\mathrm{H})$, elle affirme la prémisse mineure $(\mathrm{m})$. Les défenseurs de cette interprétation déclarent que Socrate est le vrai représentant de Platon dans le dialogue. Cela les conduit à une version restreinte de la théorie des Idées.

L'interprétation de Proclus ne coïncide avec aucune des deux précédentes. Selon Proclus, l'argument ne se veut pas une réfutation

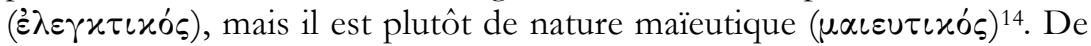
ce point de vue, le but de Parménide est d'avancer vers une meilleure compréhension de la théorie que Socrate défend et non d'attaquer la

12. Comme on le sait, les questions de savoir dans quelle mesure Aristote est un témoin fiable pour cette doctrine platonicienne et qui, de ses contemporains, pourrait avoir soutenu ces doctrines, restent débattues. Pour une discussion approfondie de ces arguments, voir Fine 1993.

13. Proclus exprime assez fréquemment cette conviction (voir p. ex. In Parm. IV, 883.8-17 et 971.22-25), qu'il reprend sans doute de ses prédécesseurs.

14. In Parm. IV, 838.19-20 et 856.25-28. Proclus développe la même approche à propos des autres apories liées à la théorie des Idées : cf. In Parm. IV, 849.2-3, 873.31-32 et 907.8-9. 
théorie elle-même. La discussion dans le Parménide est seulement une occasion pour le jeune Socrate de se former sa propre opinion à propos des limites de la théorie des Idées. Cette idée revient dans un passage où Proclus interprète le reproche que Parménide adresse à Socrate :

Par sa critique de la perplexité de Socrate, Parménide pourrait paraître à quelques-uns poser lui-même des Idées de toutes choses ( $\delta$ ó

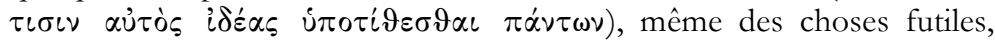
tout à fait matérielles et contre nature ; car pourquoi le critiquerait-il, si, lui, il ne rattachait pas l'existence de ces choses à la cause idéale ? Mais moi, je crois qu'il n'adresse pas cette critique à Socrate, mais à ceux qui considèrent que la génération de ces choses, en tant que futiles et viles, n'a pas de cause. Parménide rectifie cette opinion; car il n'admet en aucune façon qu'il y ait quelque chose sans cause ${ }^{15}$.

D’après Proclus, Socrate et Parménide peuvent tous deux prétendre à la vérité : le premier, parce qu'il limite avec raison la portée de l'hypothèse des Idées; le second, étant donné qu'il remarque adéquatement que même les choses qui ne sont pas causées par des Idées doivent néanmoins être causées de quelque façon. Cette approche maïeutique du passage est typique de l'exégèse proclienne et pourrait bien être une contribution originale à l'interprétation du dialogue ${ }^{16}$. L'interprétation de Proclus est toutefois moins originale quand il s'agit de la question factuelle concernant l'extension des Idées. Dans le dernier passage cité, Proclus se place lui-même parmi ces exégètes qui acceptent l'interprétation restrictive que donne Socrate de la théorie des Idées. Dans une longue digression sur le domaine des Idées, dans son Commentaire sur le Parménide, Proclus soutient qu'il y a seulement des Idées de substances naturelles et universelles, ainsi que de leurs propriétés constitutives et de leurs perfections - mais pas d'Idées d'individus, de parties, de propriétés purement accidentelles ou de choses mauvaises. Il prend aussi la peine d'expliquer comment cette doctrine est confirmée par une lecture attentive du passage dans le Parménide ${ }^{17}$. Les seules choses dont Socrate accepte des Idées dans le dialogue sont, en effet, des propriétés générales, telles que la Ressemblance et l'Unité, ou des perfections, telles que la Justice, la Beauté et le Bien. Des propriétés

15. In Parm. III, 834.26-835.7.

16. Cela est suggéré par la façon dont Proclus oppose l'interprétation maïeutique à l'interprétation de ses prédécesseurs : voir p. ex. In Parm. III, 835.3 : દ̇uoi $\delta \dot{\varepsilon} ~ \delta \circ \varkappa \varepsilon \tilde{\imath} . .$. ;

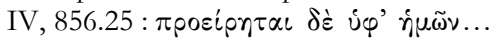

17. Pour une discussion détaillée de l'exégèse de Proclus de Parm. 129b3-e4, voir d'Hoine 2009, p. 319-325). 
purement accidentelles, il n'est pas fait mention. Par ailleurs, à propos de l'Idée de l'Homme, Socrate était resté interloqué. Proclus explique cette stupéfaction en mettant en avant une ambiguité dans le terme « homme », qui peut se référer soit à l'espèce immortelle, soit à l'individu mortel. Si l'on prend le terme dans le premier sens, Socrate a de bonnes raisons d'accepter une Idée de l'Homme, mais pas dans la seconde acception - car les Idées d'individus n'existent pas ${ }^{18}$. Finalement, Proclus considère le rejet par Socrate d'Idées du poil, de la boue ou de la saleté comme une confirmation de sa propre doctrine selon laquelle il n'y a pas d'Idées de parties du corps (poil), ni de mélanges artificiels d'éléments (boue/argile), ni du mal (saleté) ${ }^{19}$. À la fin de sa discussion sur le domaine des Idées, Proclus conclut donc comme ceci :

Ces choses étant démontrées, résumons-nous en une seule proposition générale, et disons qu'il y a des Idées des substances universelles et des perfections qui sont en elles ${ }^{20}$.

Le commentateur néoplatonicien transmet sans doute l'interprétation courante de son époque. Nous trouvons en effet la même doctrine, en des mots presque identiques, chez le maitre de Proclus, Syrianus, qui déclare en outre qu'il est lui-même largement d'accord à ce sujet avec, entre autres, Plotin et Jamblique ${ }^{21}$. Dans son Manuel de Platonisme, déjà Alcinous ( $\mathrm{II}^{\mathrm{e}} \mathrm{s}$.) dit que cette vision restrictive était la communis opinio parmi ses contemporains, et elle pourrait peut-être même remonter à l'ancienne Académie ${ }^{22}$. Il y a peu de doute que la grande majorité des platoniciens de l'époque impériale ne pensaient pas qu'il y avait des Idées de toutes choses, même s'ils n'étaient probablement pas d'accord sur tous les détails.

\section{«Ceux qui ont accepté des Idées de toutes choses»}

Et cependant cette doctrine n'était certainement pas partagée par tous les platoniciens antiques, car on trouve aussi, éparpillées à travers les pages du commentaire de Proclus sur le Parménide, des traces d'une lecture alternative du passage. À l'époque de Proclus, comme à la nôtre,

18. In Parm. III, 814.2-6.

19. In Parm. III, 832.2-10.

20. In Parm. III, 831.17-19.

21. Pour ce qui concerne Syrianus, voir In Metaph. 107.38-108.2 et 108.25-26. Pour les références aux prédécesseurs de Syrianus, voir In Metaph. 38.36-39.6 et 114.2-6.

22. Voir Alc., Didasc. IX, p. 163.23-31 et Xenocr. apud Proclum, In Parm. IV, 888.12-28 (= fr. 30 Heinze). Sur ce dernier fragment, voir p. ex. Dillon 2003, p. 118-121. 
certains exégètes de Platon doivent avoir proposé une interprétation qui donne à la théorie des Idées platonicienne une portée beaucoup plus vaste, voire universelle. Pour ceux-ci, ce n'est pas Socrate mais Parménide qui est le véritable porte-parole de Platon dans le passage. Cette interprétation étendue de la théorie des Idées est moins bien documentée dans nos sources. Prenons tout d'abord en considération en quoi cette position consiste exactement. Si quelques exégètes du Parménide étaient connus pour avoir accepté des Idées «de toutes


supposer est qu'ils aient admis des Idées correspondant à toutes les classes mentionnées par Parménide. Dès lors, nous pouvons en conclure qu'ils acceptaient des Idées non seulement des relations générales et des perfections, mais aussi des espèces naturelles, et même des choses triviales. Comme on l'a vu, relations et qualités éthiques ou esthétiques sont les exemples d'Idées dont Platon se sert le plus souvent dans les dialogues, raison pour laquelle Proclus, comme la plupart de ses prédécesseurs, pense que ces Idées ne posent aucun problème ${ }^{23}$. En outre, bien que Socrate soit resté perplexe en ce qui concerne les espèces et les éléments naturels, des Idées de ces réalités apparaissent dans d'autres dialogues et elles gagnent certainement en importance à partir de l'ancienne Académie. Dans les textes du moyen et du néoplatonisme qui ont été préservés, ce sont de telles Idées qui occupent la position centrale dans la théorie, ce qui suffit à expliquer pourquoi Proclus les accepte sans aucune réserve. Pour les Idées d'objets triviaux, en revanche, c'est tout autre chose. Elles sont rejetées de façon quasi unanime depuis Alcinous jusqu'à Syrianus et au-delà24. Cependant, dans le commentaire de Proclus, nous trouvons quelques traces d'une opinion différente, concernant ce que Parménide mentionne comme le plus vil et le plus méprisable : la saleté. Bien que la saleté n'ait, selon Proclus, pas même une existence propre, il y a dû y avoir quelque platonicien isolé pour penser autrement, car Proclus écrit ceci :

La saleté, n'ayant pas non plus de génération proéminente, a par nature une existence accidentelle. Cependant, parmi nos prédécesseurs il y en a eu un qui a essayé très énergiquement d'établir qu'il y a un paradigme de la saleté. Car s'il y a un paradigme de vertu, dit-il, alors il y en a aussi un

23. Pour un exposé plus détaillé sur les opinions des néoplatoniciens tardifs à propos des Idées de relations et de perfections, je me permets de renvoyer à d'Hoine (à paraître).

24. Voir par exemple Alc. Didasc. IX, 163.28-29 ; Plot. Enn. V, 9 [5], 14.7-11 ; Syr. In Metaph. 39.1-4 ; Procl. In Parm. III, 832.2-3. 
de la purification; et si cela est le cas, alors il y en a aussi un de la saleté ; car toute purification est une élimination de quelque saleté25.

Contrairement à son usage dans le Commentaire sur le Timée, dans son Commentaire sur le Parménide Proclus nomme rarement ses sources, ce qui rend difficile l'identification, même conjecturale, de celui qu'il critique dans le passage ci-dessus. L'argument semble basé sur le principe général que, si l'un de deux termes corrélatifs correspond à une Idée, cela doit être le cas pour l'autre également. Le cas de la saleté est donc l'application d'une règle formulée quelques lignes plus loin dans le texte, où Proclus fait à nouveau référence à l'opinion d'un philosophe anonyme : si l'Intellect connaît quelque chose de bon, il doit aussi avoir connaissance du mal correspondant ; il doit donc y avoir une Idée du mal dans l'Intellect divin ${ }^{26}$. En défendant sa position, le philosophe anonyme peut s'être reporté - comme le fait Proclus ${ }^{27}$ - au Phédon (97d1-5), où il est dit que la connaissance du meilleur et du pire coïncident. Si c'est la manière correcte de connecter les deux passages, alors la logique de l'argument rapporté pourrait être que la propreté est le bien d'un objet dont le caractère mauvais correspondant consiste à être sale. En d'autres termes, la saleté est un mal pour l'objet qui en est affecté et le processus en vue du bien correspondant - c'est-à-dire la propreté - ne peut pas être compris en dehors de sa relation à cet état déficient. Proclus luimême ne met pas en question cette connexion de la saleté avec le mal. Mais, contrairement au philosophe anonyme, il n'accepte pas d'Idées de choses mauvaises. Si Parménide reproche à Socrate, non d'avoir indûment exilé les objets triviaux du monde intelligible, mais plutôt de ne pas avoir réalisé que même ces objets ont, d'une manière ou d'une autre, une cause - ce qui est, comme nous l'avons vu, la manière dont Proclus comprend le passage -, alors le texte du Parménide confirme le rejet par Proclus d'Idées de choses mauvaises. Donc, si divergentes que puissent être leurs exégèses du passage, le philosophe anonyme et Proclus interprètent tous deux la discussion entre Socrate et Parménide comme une application de leurs propres théories respectives sur le mal.

Or, dans toute la tradition platonicienne, un seul philosophe est connu pour avoir défendu l'existence d'Idées de choses mauvaises Amélius, disciple de Plotin ${ }^{28}$. Dans un excellent article sur ce philosophe,

25. In Parm. III, 832.9-14.

26. In Parm. III, 832.26-27.

27. In Parm. III, 833.1-2.

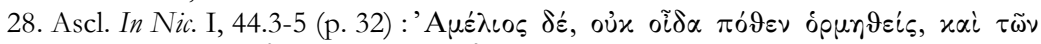

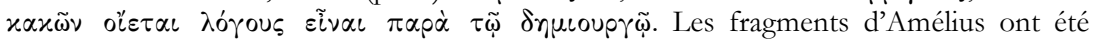
rassemblés dans Zoumpos 1956. Cependant, étant donné que le recueil de Zoumpos est 
Luc Brisson suggère qu'Amélius pourrait avoir présenté cette doctrine dans un commentaire sur la première partie du Parménide, qui était comme on le sait - le locus classicus des discussions sur le domaine des Idées $^{29}$. Cette suggestion n'est pas entièrement sans fondement, puisque nous savons qu'Amélius a aussi donné une interprétation de la seconde partie du Parménide - interprétation dont Proclus fait état dans son propre commentaire ${ }^{30}$. En outre, on sait aussi que, dans son exégèse de la première partie du Parménide, Proclus discute à plusieurs endroits des doctrines d'Amélius, toujours rapportées de façon anonyme. C'est certainement le cas, par exemple, pour la doctrine amélienne de la participation au niveau intelligible ${ }^{31}$ et peut-être aussi pour sa compréhension de la participation même $^{32}$. Enfin, dans son Commentaire sur la République (II, 275.30-276.3), Proclus affirme qu'Amélius a soutenu la thèse que la même $\pi \rho \circ \alpha i \rho \varepsilon \sigma \iota \varsigma$ est responsable aussi bien pour le vice $(\varkappa \alpha x i \alpha)$ que pour la vertu $(\alpha \rho \varepsilon \tau \dot{n})$, parce que c'est la même opération délibérative qui s'applique aux contraires. Comme nous l'avons vu, le philosophe anonyme, dans le Commentaire de Proclus sur le Parménide, a établi l'existence d'Idées de choses mauvaises ( $x \alpha x i \alpha$, dont jú $\pi \circ \varsigma$ est un exemple) en disant que la connaissance d'un de deux termes contraires implique celle de l'autre terme aussi. Puisque la suppression de la saleté (= la $x \dot{\alpha} \vartheta \alpha \rho \sigma \iota \varsigma)$ est une vertu $(\dot{\alpha} \rho \varepsilon \tau \dot{\eta})^{33}$ et relève

incomplet et ne présente pas d'apparat critique, j'ai préféré me référer aux textes originaux. Une liste étendue des fragments d'Amélius se trouve dans Brisson, 1987, p. $855-858$.

29. Brisson 1987, p. 828.

30. Cf. Saffrey-Westerink 1968, p. LXXX-LXXXI. L'attribution d'une interprétation de la seconde partie du Parménide à Amélius est basée sur deux notes marginales dans la tradition manuscrite du Commentaire sur le Parménide de Proclus : ces notes (ad In Parm. VI, 1052.25-1053.7) rendraient compte de l'interprétation des hypothèses proposée par Amélius.

31. Selon Proclus, In Tim. III, 33.33-34.3 et Syrianus, In Metaph. 109.12-13, Amélius a défendu la thèse que, même au niveau intelligible, il y a des relations de type modèleimages. Il faut sans doute comprendre cette doctrine dans le contexte de sa théorie des trois Intellects, que je présenterai plus bas. Proclus discute aussi cette thèse, erronée à son avis, dans le Commentaire sur le Parménide: voir In Parm. II, 743.18 sqq. et, dans la discussion sur le domaine des Idées, In Parm. III, 816.8-22.

32. En In Parm. IV, 839.16 sqq. Proclus discute trois métaphores d'origine platonicienne pour comprendre la participation des choses sensibles : le participant serait comme l'impression du sceau dans la cire, comme la réflexion dans un miroir, ou encore comme une image ressemblante. Ces trois métaphores ont chacune une validité restreinte. C'est la raison pour laquelle, en In Parm. IV, 846.24-26, Proclus fait référence à un platonicien anonyme, peut-être Amélius ( $c f$. Syr. In Metaph. 119.12-15), qui aurait combiné ces trois métaphores comme révélatrices de différents aspects de la participation des choses sensibles. Voir Steel 2008, p. 92.

33. Proclus utilise ces termes en In Parm. III, 832.12-14. 
donc d'une Idée, le vice qui lui correspond doit lui aussi dépendre d'une Idée. Bien que l'argument ne soit pas exactement identique, il y a de toute évidence une certaine analogie entre eux. Cela pourrait être considéré comme un indice supplémentaire qu'Amélius est ici la cible de Proclus. Si ces suppositions sont justes, il est alors raisonnable de penser qu'il est aussi le philosophe à qui Proclus fait allusion dans sa discussion sur la saleté et le mal34.

Naturellement, cela ne constitue pas une base suffisante pour affirmer que, lorsque Proclus parle des interprètes du Parménide qui ont accepté des Idées «de toutes choses ", c'est Amélius qu'il a à l'esprit. Toutefois, dans au moins deux autres passages, Proclus met en rapport l'acceptation des Idées de choses mauvaises avec le postulat de l'existence d'Idées « de toutes choses». La discussion la plus étendue chez Proclus sur les causes du mal se trouve dans son traité De l'existence du mal. Avant de présenter son propre avis sur le problème, Proclus réfute différentes explications de ses prédécesseurs. Une des opinions attaquées concerne la dérivation des choses mauvaises de paradigmes intelligibles ${ }^{35}$. Proclus discute cette doctrine en utilisant des arguments très semblables à ceux que nous trouvons dans le Commentaire sur le Parménide ${ }^{36}$. Différents interprètes pensent qu'Amélius est la cible de Proclus à cet endroit ${ }^{37}$. Ce qui doit nous intéresser ici, c'est que Proclus y appelle le(s) défenseur(s) de la doctrine selon laquelle les choses mauvaises sont causées par des Idées «ceux (...) qui placent les Idées de toutes choses dans la réalité intellective $»^{38}$. Dans le livre premier de la Théologie platonicienne, nous trouvons un parallèle très proche de ce passage. Dans une discussion sur la connaissance des dieux, Proclus écrit :

Quant à moi, je m'étonne qu'il y ait des platoniciens pour attribuer à l'Intellect la connaissance de toutes choses, je veux dire même de ce qui est individuel, contre nature et, en général, du mal, et qui de ce fait

34. Que Proclus se dispute ici vraiment avec quelqu'un en particulier et ne réfute pas simplement une position inventée est clair aussi en In Parm. III, 830.7 ( $\varepsilon \dot{\imath} \delta \dot{\varepsilon} \lambda \lambda \dot{\varepsilon} \gamma o \iota$

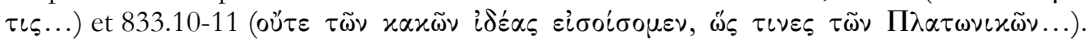

35. De mal. subs. 40.10-13 et 40.16-20, une opinion discutée davantage aux paragraphes 43 et 44 de la même œuvre.

36. Cela avait été noté aussi par les traducteurs de Proclus, De mal. subs.: voir Opsomer-Steel 2003, p. 125 n. 311.

37. Voir p. ex. Opsomer-Steel 2003, p. 124 n. 290 ; cf. aussi Erler 1978, p. 145 n. 3, où le traducteur est toutefois plus hésitant.

38. Voir De mal. subs. 40.16-17: bii quidem omnium ideas in intellectuali ponentes (le texte

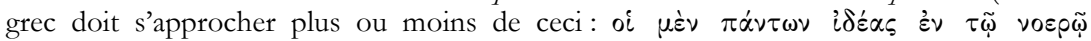
$\tau \iota \vartheta \varepsilon ́ v \tau \varepsilon \varsigma)$. 
supposent l'existence de modèles intelligibles de ces choses ellesmêmes ${ }^{39}$.

Étant donné que les choses contraires à la nature sont une sous-classe des choses mauvaises ${ }^{40}$, le(s) platonicien(s) dont il est question dans ce passage est (sont) blâmé(s) pour avoir postulé deux classes d'Idées : des individus et des choses mauvaises. Dans les annotations accompagnant leur édition de la Théologie platonicienne, Saffrey et Westerink ont mis ce passage en relation avec deux philosophes différents. Alors qu'ils pensent que c'est Amélius qui est mis en cause à propos des Idées de choses mauvaises, ils pointent du doigt Plotin quand il s'agit de l'acceptation présumée d'Idées d'individus ${ }^{41}$. Cette interprétation soulève cependant plusieurs problèmes. Tout d'abord, Proclus se réfère à un (groupe de) philosophe(s) qui accepte des paradigmes à la fois d'individus et de choses mauvaises. S'il y avait un philosophe pour soutenir l'existence d'Idées d'individus et un autre pour affirmer celle des choses mauvaises, Proclus ne pourrait pas dire qu'il y a des platoniciens qui attribuent la connaissance de toutes choses à l'Intellect. Ensuite, pour attribuer à Plotin l'acceptation d'Idées d'individus, Saffrey et Westerink font référence au traité Sur la question de savoir s'il y a aussi des Idées d'individus (V, 7 [38]). Bien qu'il soit loin d'être clair à quoi exactement les arguments de Plotin se rapportent dans ce traité aporétique, la plupart des commentateurs contemporains s'accordent pour dire que Plotin considère, dans ce texte, uniquement les Idées d'âmes (humaines) individuelles ${ }^{42}$. Or, Proclus admet, lui aussi, dans son Commentaire sur le Parménide, des Idées de certaines âmes individuelles ${ }^{43}$. Il serait dès lors étrange de supposer qu'il critique Plotin pour une doctrine qu'il a lui-même défendue ailleurs. En outre, dans un contexte très similaire, dans son Commentaire sur la Métaphysique d'Aristote, le maitre de Proclus, Syrianus, compte Plotin parmi ceux de ses prédécesseurs qui nient, entre autres choses,

39. Theol. plat. I, \21, p. 98.16-20 (trad. Saffrey-Westerink) ; cf. I, \18, p. 87.22-24 et les notes ad locos (p. 153 et 156).

40. Cf. De mal. subs. 55.1-14, où trois types de mal sont distingués; être «contraire à la nature » est le type de mal le plus bas, qui se produit dans les corps. Je crois qu'un bon exemple d'une telle forme de mal serait l'existence parasitique de la saleté. Il convient de

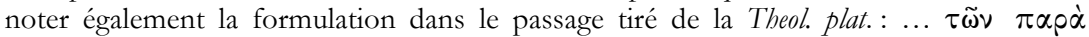

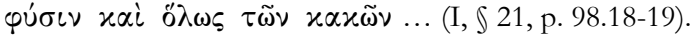

41. Saffrey-Westerink 1968, p. 153 n. 2 ad 87 ; p. 156 n. 3 ad 98.

42. Voir récemment p. ex. Gerson 1994, p. $72-78$ et Kalligas 1997, p. 211 sqq. Les textes et la bibliographie pertinents sur ce sujet sont maintenant rassemblés dans Sorabji 2004, p. 262-267.

43. Pour la doctrine de Proclus à propos des Idées d'âmes, voir In Parm. III, 817.4819.21 . 
l'existence d'Idées d'individus ${ }^{44}$. Cela suggère que, dans leur critique des Idées d'individus, Syrianus et Proclus ne se préoccupent pas des âmes individuelles (pour lesquelles ils acceptent des Idées), mais des êtres vivants particuliers. En effet, Plotin également refuse les Idées de ce genre d'individus - pas dans Ennéades V, 7 [38], mais dans le traité V, 9 [5], auquel Syrianus fait référence explicitement ${ }^{45}$. Il serait difficile de comprendre pourquoi Proclus tiendrait ici des propos contraires aux enseignements de son maitre sur le même sujet, surtout parce que les deux commentateurs s'accordent en général sur toutes les questions concernant l'extension des Idées et parce que leurs discussions dérivent, comme je l'ai montré ailleurs, d'une source commune ${ }^{46}$. Je voudrais dès lors tenter de proposer une autre solution à l'énigme sur l'identité du philosophe anonyme dans le Commentaire de Proclus sur le Parménide. Ne pourrait-il s'agir d'Amélius ? Si cette hypothèse s'avère plausible et si, comme le suggère le passage cité plus haut, les choses mauvaises et les individus sont vraiment les dernières choses qu'un platonicien orthodoxe mettrait en rapport avec les Idées, il est alors naturel de supposer que, parmi les platoniciens de l'antiquité tardive, Amélius était réputé avoir accepté des Idées «de toutes choses». Et où aurait-il pu avoir une meilleure occasion de proposer une telle doctrine que dans son Commentaire présumé sur le Parménide?

\section{Proclus contre les Idées d'individus}

Notre tâche consiste donc à reconstituer la doctrine d'Amélius à propos des Idées d'individus. Avec cette question à l'esprit, nous devons nous tourner d'abord à nouveau vers Proclus. Dans son Commentaire sur le Parménide, le néoplatonicien athénien a inséré une longue digression sur le domaine des Idées, qui est notre source principale pour la reconstruction de ce débat dans l'antiquité tardive ${ }^{47}$. L'une des difficultés discutées par Proclus concerne les Idées d'individus. Qu'il n'y a pas d'Idées d'individus est pour Proclus hors de doute. Dans ce rejet, il est en bonne compagnie platonicienne, puisqu'au moins Alcinous, Plotin, Jamblique et Syrianus ont partagé cette opinion ${ }^{48}$. Quoi qu'il en soit, il

44. Cf. Syr. In Metaph. 38.36-39.6.

45. Voir Plot. Enn. V, 7 [5], \$ 12.

46. D'Hoine 2009, p. 311-342.

47. Pour cette digression, voir In Parm. III, 815.11-831.16. La discussion de Proclus sur le domaine des Idées commence en In Parm. 805.2 par une exégèse de Parm. 130b3-5 et continue jusqu'à la fin du livre III.

48. Pour Alcinous, voir Didasc. IX, 163.27-28; pour les opinions de Plotin et de Jamblique telles que Syrianus les rapporte, voir In Metaph. 38.37-39.4 ; pour Syrianus luimême, voir aussi 107.12-14. 
convient de savoir de quelle sorte d'individus il est question dans la digression que Proclus leur consacre. Celui-ci ne se préoccupe pas des Idées d'âmes individuelles, dont - comme nous l'avons vu - il a déjà traité dans une section précédente de son commentaire. Le problème auquel Proclus doit faire face concerne une réalité composée (un

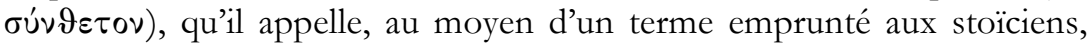

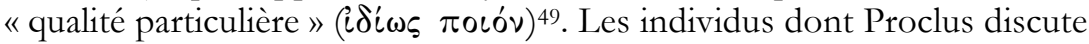
sont les membres particuliers des espèces naturelles (plantes et animaux), qui ont une existence temporaire dans le monde sensible en tant que composés de forme et de matière. Chaque individu est numériquement distinct et qualitativement différencié de tous les autres membres de la

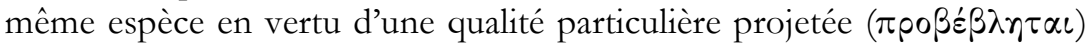
dans un corps sensible. Selon Syrianus, nous devrions dans chaque cas faire une distinction entre la qualité en vertu de laquelle un être

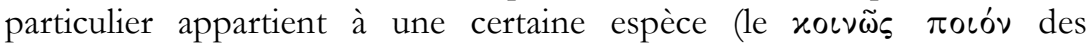

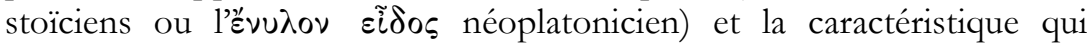
intervient en plus de la première et qui, différente pour chaque individu

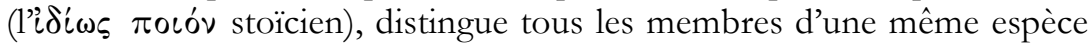
les uns des autres. Aucune de ces deux caractéristiques ne coïncide avec le corps; cependant, elles périssent toutes deux avec lui ${ }^{50}$. La caractéristique individuelle ne coïncide pas non plus avec la vraie personne, qui est notre âme, bien que la vie de l'âme sur terre soit déterminée par elle ${ }^{51}$.

Dans sa discussion sur les Idées d'individus, Proclus avance trois arguments contre la thèse que l'individualité ainsi conçue trouve son origine dans les Idées transcendantes ${ }^{52}$. La peine qu'il prend pour réfuter cette position erronée semble suggérer qu'il est impliqué dans une discussion réelle avec l'un de ses prédécesseurs et qu'il n'insiste pas

49. À propos de l'utilisation de ce terme par Proclus, voir In Parm. III, 824.11 et V, 981.8-9. Même si ce terme stoïcien a été associé depuis Porphyre avec la théorie de l'individu comme résultant d'un «bundle of qualities » unique, Proclus critique ailleurs une telle théorie de l'individualité. Cf. Procl. apud Ol., In Alc. 204.8-12, où Proclus mentionne le «Peripatos », bien que sa critique soit probablement dirigée contre Porphyre : voir Chiaradonna 2000, p. 326 n. 58.

50. Cf. Syr. In Metaph. 28.11-29.11. Sur ce passage, voir aussi Longo 2005, p. 386-388.

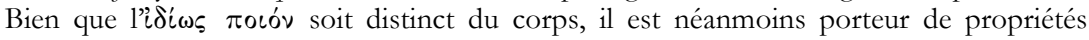

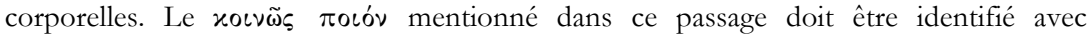

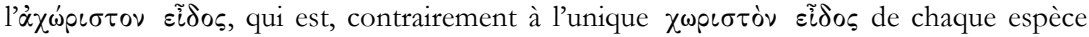

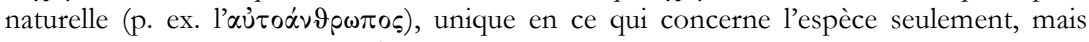
numériquement multiple ou même infini : cf. Syr., In Metaph. 39.18-20.

51. La vraie personne est son âme rationnelle: voir Procl. apud Ol. In Alc. 203.20204.8 ; cf. In Tim. III, 330.19-24. Cf. Opsomer 2006, p. 162-166.

52. Proclus discute le problème des Idées d'individus en In Parm. III, 824.9-825.7. 
seulement sur un topos hérité de la tradition. En effet, nous avons vu que, dans la Théologie platonicienne, Proclus fait référence à des philosophes anonymes qui attribuent une connaissance de toutes les choses, y compris des individus, à l'Intellect. Et quand Syrianus nous dit que « les

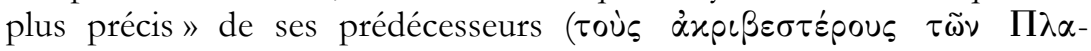
$\tau \omega \nu\llcorner\varkappa(\widetilde{\omega})$ ) n'acceptaient pas d'Idées d'individus, il sous-entend (ou du moins nous pouvons le supposer) par là que certains moins grands esprits le faisaient ${ }^{53}$. Mais examinons d'abord les trois contre-arguments de Proclus.

(1) [S]i tout ce qui vient à l'être selon une Idée vient à l'être selon une cause immuable, et si tout ce qui doit son existence à une cause immuable est par son essence inaltérable, Socrate et chacun des individus sera par son essence toujours le même et fondé éternellement. Mais cela est impossible ${ }^{54}$.

Le premier argument est basé sur un principe néoplatonicien bien connu: tout ce qui vient à l'être par une cause immobile est nécessairement exempt des changements qui se produisent au cours du temps ${ }^{55}$. Puisque les Idées sont des causes immobiles, tandis que les individus naissent et meurent, ces derniers ne peuvent pas être causés par celles-là. Proclus prend cette règle comme un principe général dans ses discussions à propos du domaine des Idées. Elle sert de preuve pour l'existence des Idées d'espèces naturelles et de critère principal dans la discussion des Idées d'âmes rationnelles et irrationnelles et de natures ${ }^{56}$. Déjà dans les Éléments de théologie, Proclus avait utilisé ce principe comme un argument contre les Idées d'individus ${ }^{57}$. Étant donné que Proclus ne fait qu'appliquer ici un principe général de sa métaphysique, ce premier argument n'est pas nécessairement dirigé contre quelqu'un en particulier. Passons donc au deuxième argument.

(2) De plus il est absurde que l'Idée soit tantôt le paradigme d'une chose, tantôt ne le soit pas. Car ce qui est éternellement possède

53. Syr. In Metaph. 108.12-13.

54. Procl. In Parm. III, 824.12-16.

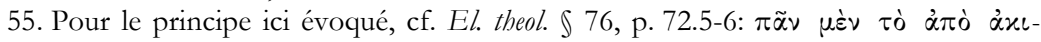

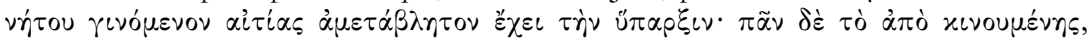
$\mu \varepsilon \tau \alpha \beta \lambda \eta \tau \dot{v}$. Dans son commentaire ad locum (p. 241), Dodds fait référence à In Tim. I, 294.12-14, où Proclus attribue l'invention de cette doctrine à Aristote. Déjà Syrianus utilise cet argument contre l'existence d'Idées d'individus : voir Syr. In Metaph. 107.12-14.

56. Voir In Parm. III, 824.4-8 (espèces naturelles), 817.19-20 (âmes rationnelles), 819.23-26 (âmes irrationnelles), et 821.16-19 (natures).

57. Voir El. theol. $\$ 178$ et Dodds 1963 ad locum (p. 293). 
éternellement tout ce qu'il possède, et par conséquent ou n'aura pas l'Idée ou l'aura toujours : dire en effet que dans les intelligibles il se produit quelque chose par accident est absurde. Si donc le paradigme sera éternel, l'image doit être éternelle aussi, car chaque paradigme est le paradigme d'une image. Or si la chose qui existe en accord avec lui tantôt est, tantôt n'est pas, le paradigme aussi sera tantôt paradigme, tantôt ne le sera pas, et il le sera dans une courte partie du temps, mais pas dans le temps infini. Or le «est» et le «n'est pas» alternativement est une chose complètement étrangère à cette substance ${ }^{58}$.

Ce deuxième argument s'appuie sur le statut des Idées en tant que paradigmes. Proclus considère les Idées d'abord et avant tout comme des causes paradigmatiques, à la ressemblance desquelles le démiurge forme le monde sensible ${ }^{59}$. Cette insistance sur le statut paradigmatique des Idées, qui rappelle le rôle du Paradigme intelligible dans le Timée de Platon, est assez commune au moins à partir du moyen platonisme ${ }^{60}$. Or, si les Idées sont des modèles et si un modèle est toujours le modèle d'une image ${ }^{61}$, alors - dit Proclus - il s'ensuit nécessairement que les Idées ont des images. De plus, si les Idées sont des modèles éternels, ce dont chaque Idée est un modèle doit aussi résister à la corruption. Il n'est pas possible qu'une Idée qui existe pour une période de temps infinie

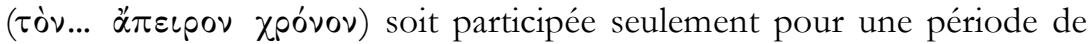
temps limitée. Or, puisque cela est exactement ce qui arriverait si nous acceptions des Idées d'individus, il faut rejeter de telles Idées. Apparemment, cet argument s'adresse à quelqu'un qui était enclin à accepter que les Idées ne soient pas nécessairement toutes toujours

58. Procl. In Parm. III, 824.16-24.

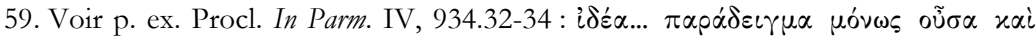

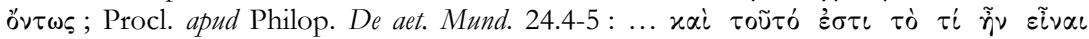

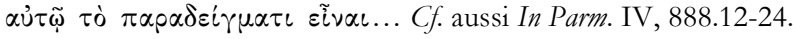

60. Voir Plat. Tim. 30c2-31b3 ; cf. aussi Ar. De Id. 83.19 et 86.15. Le caractère paradigmatique des Idées est mis en évidence dans la célèbre définition médioplatonicienne des Idées comme "causes paradigmatiques des choses naturelles ", qui - d'après Proclus, In Parm. 888.12-15 - remonte à Xénocrate (fr. 30 Heinze). Pour les variantes de cette définition chez des auteurs postérieurs, voir Ar. Did. Ep. Fr. Phys. 1 Diels ; Sen. Ep. LVIII 19.2-3 ; Alc. Didasc. 163.23-24 ; Diog. Laert. III, 77, 12-14 ; Calc. In Tim. \304, p. 306.6-7 (cf. \$330, p. 324.15-16).

61. Selon Proclus, c'est le Timée de Platon qui nous enseigne que le modèle est toujours modèle d'une image (voir In Parm. IV, 911.1-3), mais la référence est plutôt générale. Aristote était probablement le premier à mettre en évidence cette relation

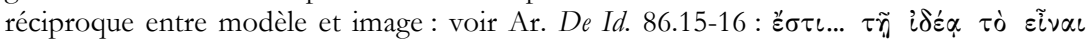

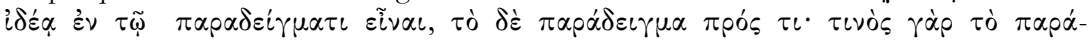
$\delta \varepsilon \iota \gamma \mu \alpha \pi \alpha \rho \alpha ́ \delta \varepsilon \iota \gamma \mu \alpha$. 
participées. Avant d'essayer d'identifier cet auteur, nous devons encore examiner le dernier argument de Proclus contre les Idées d'individus.

(3) Comment donc ne pas croire Socrate, quand il dit que nous sommes contraints de poser les Idées pour cette raison, à savoir, d'avoir l'un avant les plusieurs. Car s'il y a des Idées même des individus, il y aura un un avant l'un, ou plutôt une infinité de choses seront avant les choses numériquement finies; car les Idées seront infinies, tandis que les choses qui sont dans le sensible sont finies. Or que peut-il y avoir de plus absurde que cela ${ }^{62}$ ?

Pour un lecteur moderne, l'objection la plus évidente contre les Idées d'individus est sans aucun doute le fait que les Idées ont été introduites pour expliquer l'unité dans la pluralité, c'est-à-dire pour fournir un point de référence unique et stable qui puisse servir pour plusieurs instanciations particulières de la même propriété. Le dernier argument que Proclus avance contre l'existence d'Idées d'individus se place dans cette perspective. À n'importe quel moment dans le temps, le nombre d'êtres individuels dans le monde sensible est limité. Or, si nous acceptions une Idée pour tous les individus et si les Idées sont éternelles, il devrait aussi y avoir des Idées pour des choses particulières qui n'existent pas encore et pour celles qui n'existent plus. Parce que le temps est infini, le nombre d'Idées que nous devrions alors postuler non seulement dépasserait le nombre de choses sensibles particulières existant à n'importe quel moment dans le temps, mais il serait même infini $\left(\alpha^{\prime} \pi \varepsilon \iota \rho \alpha\right)$, ce qui est totalement absurde ${ }^{63}$. Puisque la priorité ontologique implique un degré supérieur d'unité, ce qui est numériquement illimité ne peut jamais être la cause d'un nombre limité d'objets. Dans son Commentaire sur le Parménide, Proclus met continuellement en garde à propos du fait que l'acceptation des Idées d'individus mènerait à une infinité numérique des Idées $^{64}$. Cet argument est donc clairement dirigé contre ceux qui étaient disposés à accepter un nombre infini d'entités dans le monde intelligible.

Les deuxième et troisième arguments peuvent nous fournir un indice quant à l'identité de celui à qui s'adressent les critiques de Proclus. Certains interprètes ont, cette fois encore, suggéré le nom de Plotin, auteur du traité intitulé Sur la question de savoir s'il y a aussi des Idées

62. Procl. In Parm. III, 824.24-825.5.

63. Qu'il ne peut pas y avoir un nombre infini d'Idées est confirmé dans El. theol. $\int 86$ et 179 .

64. Voir p. ex. In Parm. IV, 885.17-21 ; 890.12-15. 
d'individus (V, 7 [18] ${ }^{65}$. Toutefois, comme nous l'avons vu, Plotin semble accepter, dans ce traité, des Idées d'âmes individuelles, mais pas d'individus composites animés par elles. Ce qui permet que différentes incarnations de la même âme résultent en différentes personnes, ce sont

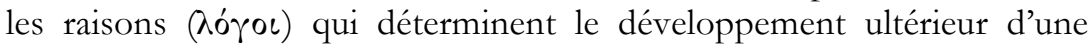
Idée durant sa descente vers les réalités inférieures de l'être. L'une des difficultés mises en avant par Plotin est précisément le fait que, si nous acceptions des Idées d'individus, nous introduirions une infinité $(\dot{\alpha} \pi \varepsilon \iota \rho \imath \alpha)$ dans la réalité intelligible ${ }^{66}$. Si le temps et le nombre d'êtres individuels sont tous deux infinis, alors accepter une Idée pour absolument chaque individu revient à postuler une infinité numérique d'Idées. Plotin évite cette conclusion en acceptant la théorie (stoïcienne) de la succession des cycles cosmiques, dans laquelle tous les événements se répètent éternellement ${ }^{67}$. Dans chaque cycle, la même âme peut animer différents corps. S’il y a une Idée pour chaque âme particulière, il n'est pas nécessaire qu'il y ait une Idée pour chaque individu. Différents individus sont engendrés par différents logoi, mais plusieurs logoi peuvent se développer à partir d'une seule Idée dans différents cycles. En outre, dans le traité Sur l'Intellect, les Idées et l'Être ${ }^{68}$, où il examine l'extension des Idées dans un contexte plus large, Plotin repousse de façon assez directe les Idées d'individus. Dans ce texte, il est clair qu'il est question d'individus «composés $»^{69}$. C'est sur la base de ce traité que Syrianus pouvait compter Plotin parmi les philosophes qui rejettent les Idées d'individus. Donc, si Plotin pose la même objection que Proclus contre les Idées d'individus, s'il accepte seulement des Idées d'âmes particulières - comme Proclus le fait aussi - et s'il est considéré par Syrianus comme quelqu'un qui nie les Idées d'individus, il paraît peu probable que les critiques de Proclus soient dirigées contre lui.

\section{Amélius sur les individus}

La solution pourrait venir une fois de plus du maitre de Proclus, Syrianus. Dans un passage remarquable de son Commentaire sur la Métaphysique, Syrianus répond à la critique d'Aristote contre ceux qui identifient les Idées avec des nombres. Si les Idées sont des nombres,

65. Voir p. ex. Morrow-Dillon 1987, p. 153 et p. 185 n. 30.

66. Voir Plot. Enn. V, 7 [18], 1.12-14, 1.25-26 et 3.22-23. Dans Enn. VI, 5 [23], 8.3942, Plotin dit clairement que le nombre d'Idées doit être fini.

67. Voir p. ex. Plot. Enn. V, 7 [18], 3.13-18.

68. Plot. Enn. V, 9 [5], 12.

69. Cf. Enn. V, 9 [5], 12.5-11, où Plotin parle de qualités corporelles, comme le nez crochu ou camus, et des couleurs. 
affirme Aristote, alors l'infini également doit être une Idée. Dans ce contexte, Syrianus écrit ceci :

Contre cette argumentation aussi ceux qui défendent la thèse suivant laquelle le nombre des modèles est infini ( $\tau \tilde{\eta} s$ à $\pi \varepsilon i \rho i \alpha \varsigma$ $\tau \tilde{\omega} \nu$ $\pi \alpha \rho \alpha \delta \varepsilon \tau \gamma \mu \alpha \dot{\alpha} \tau \omega \nu)$ soutiennent que ce n'est pas en même temps que le monde sensible reçoit la totalité des Idées; et certains d'entre eux déclarent que, même dans un temps infini, le monde ne reçoit pas les images de toutes les causes idéelles. Telle est en effet la thèse qu'ose soutenir dans son audace juvénile une personne comme Amélius, le

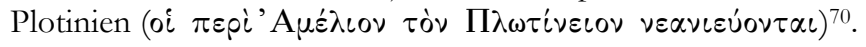

Apparemment, Amélius pense que les Idées existent en nombre infini, même si cette infinité ne sera jamais entièrement réalisée dans le monde sensible. Cela implique dès lors qu'il souscrit aux deux affirmations suivantes: (1) il y a un nombre infini d'Idées ${ }^{71}$, et (2) il y a des Idées qui ne sont pas toujours participées - certaines ne le seront en fait jamais. Pour Syrianus, comme pour Proclus, ces deux thèses sont inacceptables ${ }^{72}$. Or, les troisième et deuxième arguments de Proclus contre l'existence d'Idées d'individus sont précisément dirigés contre ces deux thèses. Cela peut-il être une coïncidence ?

Amélius Gentilianus est connu pour quelques innovations dans la compréhension néoplatonicienne de l'Intellect ${ }^{73}$. Pour expliquer l'organisation interne du monde intelligible, Amélius, développant l'interprétation par Plotin de Timée 39e dans Enn. III, 9 [13], \1, divisait l'intelligible en trois intellects démiurgiques - ou peut-être plutôt en trois aspects de l'intellect : celui qui est, celui qui $a$, et celui qui voit $t^{7}$. À ces trois aspects de l'Intellect correspondent trois états dans le déploiement des Idées. Le Vivant intelligible du Timée, qui se trouve dans le premier

70. Syr. In Metaph. 174.2-6 (trad. Brisson légèrement modifiée).

71. Cf. aussi Sen. Ep. LVIII, \18, qui appelle les Idées « innombrables».

72. Si l'on peut considérer les Idées infinies de quelque manière, c'est par rapport à leur pouvoir, pas à leur nombre, car une infinité numérique ne peut jamais exister en acte : cf. les trois sens d'infinité distingués en In Tim. III, 453.15-21; cf. aussi In Parm. II, 752.1-2. Il est d'ailleurs, comme on a vu, inconcevable que les Idées, qui doivent être plus unifiées que les êtres sensibles causés par elles, existent en nombre infini, comme Amélius le pense : cf. In Tim. III, 102.23-27 et les passages cités plus haut, aux n. 63-64. De plus, la totalité des Idées doit être participée à chaque moment dans le temps : pour Syrianus, c'est la raison pour laquelle les espèces naturelles ne peuvent jamais périr : voir In Metaph. 120.4-8.

73. Pour la doctrine amélienne de l'Intellect et la question délicate de sa relation aux doctrines de Numénius, Plotin et Porphyre, voir Brisson 1987, p. $831-836$; Dillon 1969 ; Massagli 1982 ; Corrigan 1987.

74. Procl. In Tim. I, 306.1-3 et III, 103.18-23. 
démiurge, se divise davantage dans les deux états consécutifs, le dernier étant celui dans lequel les Idées sont divisées «en individus» ( $\varkappa \alpha \vartheta^{\prime}$ ย̌ $\left.\varkappa \alpha \sigma \tau \alpha\right)$. C'est ce troisième et dernier démiurge qui exerce l'activité proprement démiurgique, puisqu'il est celui qui "crée avec ses mains » $(\mu \varepsilon \tau \alpha \chi \varepsilon \iota \rho \eta \dot{\sigma \varepsilon \iota} \pi \circ \iota \tilde{\omega} \nu)^{75}$ : il est directement impliqué dans la constitution du monde sensible.

Dans son article sur Amélius, déjà cité, Luc Brisson met en rapport le fragment, rapporté plus haut, concernant l'infinité des paradigmes, avec la théorie amélienne de l'âme ${ }^{76}$. Selon cette interprétation, le nombre infini d'Idées seraient les Idées des âmes particulières dans le troisième Intellect ${ }^{77}$. Puisque Amélius est supposé avoir accepté la doctrine platonicienne de l'immortalité de l'âme, le fait qu'il admette un nombre infini d'Idées d'âmes particulières pourrait alors suggérer que certaines âmes puissent, à un certain moment, échapper définitivement au cycle des réincarnations ${ }^{78}$. Il faut comprendre en effet que la possibilité que des âmes évitent de nouvelles incarnations implique la nécessité d'un nombre infini de celles-ci, car, si le temps est infini, de nouvelles âmes doivent remplir les vides laissés par les âmes entièrement purifiées. Et, de fait, cette doctrine entraîne que, s'il existe un paradigme pour chaque

\section{Procl., In Tim. I 361.26 ff.}

76. Voir Brisson 1987, p. 834-835. Brisson donne encore une interprétation alternative du passage : le nombre infini des Idées pourrait être le résultat de la participation des Intellects inférieurs à ceux qui leur sont supérieurs. Pourtant, je ne vois pas comment la multiplication d'un nombre limité d'entités pourrait résulter dans une infinité numérique. Une meilleure explication serait peut-être celle que Proclus présente en In Parm. II, 751.10-752.16. Proclus y discute la thèse selon laquelle chaque Idée comporte toutes les autres; mais alors les Idées dont chaque Idée est composée doivent elles aussi comporter toutes les autres Idées; et cela de nouveau pour chaque Idée faisant partie de ces Idées etc. La conséquence serait donc que les Idées existeraient en nombre infini. Proclus en tire la conclusion qu'alors même pour chaque espèce naturelle il y aurait une infinité de paradigmes (p. ex. un nombre infini d'Idées de l'Homme, correspondant au nombre infini des hommes sensibles), c'est-à-dire qu'il y aurait une Idée pour chaque individu, ce qui est absurde à son avis.

77. Si la terminologie de In Tim. I, 309.18 est celle d'Amélius, il appelait le troisième Intellect «source des âmes» ( $\pi \eta \gamma \dot{\eta} \psi u \chi \tilde{\omega} \boldsymbol{\nu})$. Cependant, Proclus semble emprunter cette expression plutôt à Théodore d'Asiné, qui a défendu une théorie semblable à celle d'Amélius.

78. Cf. Brisson 1987 p. 835 et 847. Brisson cite Ol. In Phd. 10, J 1.1-5 (p. 137 Westerink), selon lequel la théorie de la métempsychose est une conséquence inévitable de la combinaison de deux thèses: (1) celle de l'éternité du monde et (2) celle de l'immortalité de l'âme. Car seule l'hypothèse de la métempsycose peut en ce cas éviter que le nombre d'âmes soit infini. Or, vu qu'Amélius a, en bon platonicien, sans doute accepté ces deux thèses, et vu qu'il a en toute vraisemblance aussi adhéré à la théorie de la métempsycose, il ne reste qu'à supposer que selon lui certaines âmes pourraient échapper au cycle des réincarnations. 
âme particulière, alors le nombre d'Idées sera infini. Toutefois, je ne pense pas que cela soit la meilleure manière d'expliquer la position d'Amélius. Car, si l'âme est immortelle, elle devrait - à tout le moins d'après la théorie de la participation entre les différents niveaux de l'être telle qu'elle est présentée par Syrianus et Proclus - éternellement participer à son paradigme, et cela que l'âme soit elle-même incarnée ou non. Et si c'est le cas, alors aucun paradigme ne manquera de participant à aucun moment dans le temps. Or, on dit qu'Amélius a soutenu la doctrine selon laquelle certaines Idées ne seront jamais participées. Dès lors, les participants dont il est question ne peuvent pas être des âmes.

La question est maintenant de savoir s'il y a des éléments positifs pour indiquer que le nombre infini de participants aux Idées sont en fait des individus sensibles. Quelques passages au moins pourraient être interprétés dans ce sens. En Timée 30c2-7, Platon parle du modèle intelligible à la ressemblance duquel les êtres vivants du monde sensible

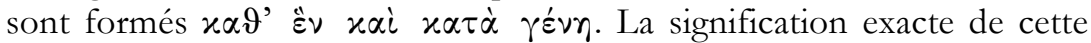
formule doit avoir fait l'objet de nombreuses discussions parmi les anciens interprètes du Timée, comme la doxographie de Proclus le suggère ${ }^{79}$. Proclus écrit :

Certains $(\tau \iota \nu \varepsilon \varsigma) \ldots$ disent que, par cette expression, ce sont aussi les individus ( $\left.\tau \dot{\alpha} \quad x \alpha \vartheta^{\prime} \tilde{\varepsilon}^{\prime} \varkappa \alpha \sigma \tau \alpha\right)$ eux-mêmes qui sont opposés aux espèces

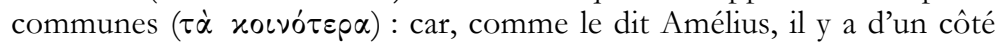
les modèles des individus ( $\tau \tilde{\omega} \nu \mu \varepsilon \rho\llcorner\chi \tilde{\omega} \nu)$, de l'autre les modèles des

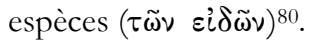

Ici encore, Brisson pense que les individus en question sont des âmes particulières ${ }^{81}$. Mais, dans ce passage, Proclus discute non la façon dont les âmes sont engendrées, mais la question de savoir comment les êtres vivants sont faits à la ressemblance du Vivant intelligible, le premier et unique paradigme de toute la création. Il vaut la peine de noter que Plotin et Proclus renvoient tous deux à ce passage dans leur discussion sur les Idées d'êtres vivants ${ }^{82}$. Puisque Amélius a identifié le Vivant avec

79. In Tim. I, 425.11-427.2.

80. In Tim. I, 425.16-19 (trad. Festugière légèrement modifiée). Cf. aussi In Tim. II, 154.4-9, où non pas Amélius, mais Théodore d'Asiné - qui est, d'après In Tim. I, 425.1922, du même avis en ces matières qu'Amélius - est mentionné.

81. Brisson 1987, p. 833, qui cite aussi In Tim. I, 309.19-20.

82. En ce qui concerne Proclus, voir In Parm. III, 823.16-18, où il cite Tim. 30d1; pour Plotin, voir Enn. VI, 7 [38], 11.2-3. 
le premier Intellect ${ }^{83}$, c'est évidemment cet Intellect qui devient de plus en plus divisé dans les Intellects inférieurs, qui prennent part au Paradigme. Amélius semble avoir pensé que le premier Intellect était

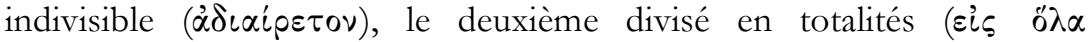

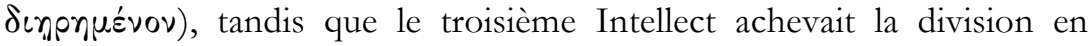
individus ( toute la création comprend de façon unitaire tous les êtres vivants, il va de soi que les «totalités » du deuxième Intellect sont les espèces d'êtres vivants et les «individus» du troisième Intellect les êtres vivants individuels.

On pourrait, bien entendu, objecter que, dans le Timée, le démiurge délègue la tâche de produire des individus aux jeunes dieux, qui engendrent les êtres mortels. C'est exact. Proclus, par exemple, fait de ce point de vue une distinction, tout comme son maître Syrianus le faisait, entre deux « démiurgies » différentes, chacune d'elles étant subdivisée en deux moments ${ }^{85}$. La première, conduite par le démiurge du Timée et par la triade hypercosmique à laquelle il préside, est la cause universelle des

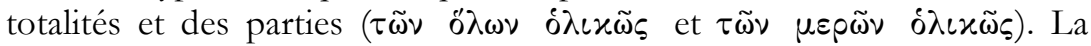
seconde démiurgie, amenée par une monade encosmique et une triade subordonnée - faisant toutes partie des «jeunes dieux » du Timée - est la cause partielle des totalités et des parties ( $\tau \tilde{\omega} \nu$ ő $\lambda \omega \nu$ ó $\lambda \iota x \tilde{\omega} \varsigma$ et $\tau \tilde{\omega} \nu$ $\mu \varepsilon \rho \tilde{\omega} \nu$ ó $\iota \iota x \tilde{\omega} \varsigma)$. Les jeunes dieux produisent les mêmes choses que le démiurge universel, mais d'une façon différente, c'est-à-dire pas comme des espèces, mais en tant qu'êtres individuels mortels, et en tant que composés de parties. Le point important ici est que Proclus présente cette doctrine de Syrianus comme une alternative à la théorie amélienne des trois démiurges ${ }^{86}$. Cela semble suggérer que les tâches que Syrianus distribue entre ses différentes monades et triades démiurgiques sont supposées être également remplies par les trois Intellects démiurgiques d'Amélius. Si c'est le cas, alors nous avons ici une confirmation indirecte que les individus produits par le troisième Intellect amélien sont des êtres vivants individuels. Malheureusement, nous ne disposons d'aucune indication quant au rôle assigné par Amélius aux jeunes dieux, ni sur la façon dont ils étaient censés être en rapport avec les trois démiurges. Il n'est cependant pas du tout exclu qu'Amélius ait considéré les parties mortelles des êtres vivants comme des produits des jeunes dieux. Les

83. Voir In Tim. III, 103.18-23. Cf. aussi In Tim. I, 309.23-24 (le premier Intellect est

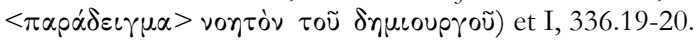

84. In Tim. I, 309.18-20.

85. Voir In Tim. I, 310.3 sqq. Pour un bon exposé de cette doctrine assez compliquée, voir Opsomer 2003, p. 9-11.

86. La théorie d'Amélius a été examinée juste avant, dans In Tim. I, 309.14-310.2. 
jeunes dieux peuvent toujours être tenus responsables de la création des corps sensibles et même des parties irrationnelles de l'âme. La seule chose qu'Amélius doit avoir affirmée en ce qui concerne le troisième Intellect est qu'il contenait un nombre infini d'Idées à partir desquelles les êtres naturels individuels pouvaient se développer. Cela signifie tout simplement que ni la matière ni le corps sensible ni n'importe quel autre produit des jeunes dieux ne peut être le principe d'individuation des individus sensibles, puisque le troisième démiurge dispose déjà de ces principes individuants. Peut-être Amélius pensait-il que les logoi que Plotin tenait pour responsables de l'individuation des âmes particulières en individus sensibles étaient déjà présents dans le troisième démiurge. Mais cela ne peut faire l'objet que d'une pure spéculation ${ }^{87}$.

Bien entendu, l'objection la plus obvie à cette interprétation reste qu'il est à peine concevable qu'un platonicien comme Amélius puisse postuler des Idées séparées pour tous les individus. La réponse est, je pense, que c'est en effet tout aussi inconcevable pour Proclus et la plupart de ses prédécesseurs néoplatoniciens, mais que, néanmoins, ils nous disent que quelqu'un s'est tout de même aventuré à défendre cette doctrine. Somme toute, est-ce vraiment plus problématique que cette autre doctrine étonnante qu'Amélius a soutenue contre la communauté orthodoxe néoplatonicienne : l'existence des Idées de choses mauvaises? En tout cas, la conclusion qui suit naturellement de toutes ces considérations est qu'Amélius était très probablement l'un des philosophes qui, d'après Proclus, admettaient des Idées de «toutes choses ».

87. Reste encore une autre question: vu qu'Amélius semble aussi avoir accepté la doctrine de la métempsycose (cf. supra, n. 78), on se demande quel rapport avaient dans sa théorie les âmes individuelles et les individus sensibles. 


\section{BIBLIOGRAPHIE}

ALLEN, R.E. 1997 : Plato's Parmenides, transl. with comment, New Haven-Londres, revised ed., 1997 (The Dialogues of Plato, vol. 4).

Brisson, L. 1987 : «Amélius : sa vie, son œuvre, sa doctrine, son style », dans : W. Haase (éd.), Aufstieg und Niedergang der Römischen Welt (ANRW), Band II 36.2, Berlin, 1987, p. 793-860.

— 1994 : Platon, Parménide, trad. inédite, introduction et notes, Paris, 1994 (GF).

Chaignet, A.-E. 1900-1903 : Proclus le Philosophe, Commentaire sur le Parménide, suivi du Commentaire anonyme sur les VII dernières hypothèses, traduit pour la première fois en français et accompagné de notes, d'une table analytique des paragraphes et d'un index étendu, 3 vol., Paris, 1900-1903.

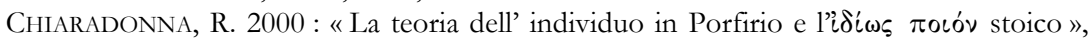
Elenchos, 21 (2000), p. 303-331.

Cornford, F.M. 1939: Plato and Parmenides: Parmenides' Way of Truth and Plato's Parmenides, transl. with an Introduction and a running Commentary, Londres, 1939 (International Library of Psychology, Philosophy and Scientific Method).

Corrigan, K. 1987 : «Amelius, Plotinus and Porphyry on Being, Intellect and the One. A Reappraisal », dans: W. Haase (éd.), Aufstieg und Niedergang der Römischen Welt ( $A N R W$ ), Band II 36.2, Berlin, 1987, p. 975-993.

Coxon, A.H. 1999: The Philosophy of Forms: An Analytical and Historical Commentary on Plato's Parmenides, Assen, 1999.

D'Hoine, P. 2008 : «The Intelligent Design of the Demiurge. On an Argument From Design in Proclus », Études platoniciennes, 5 (2008), p. 63-90.

— 2009 : «Le Commentaire de Proclus sur le Parménide comme source du 'Peri tôn ideôn logos' de Syrianus ? ", dans: A. Longo (éd.), Syrianus et la métaphysique de l'Antiquité tardive. Actes du Colloque international (Université de Genève, 29 septembre-1er octobre 2006), Naples, 2009 (Elenchos, 51), p. 311-342.

- (à paraittre) : "Forms of symbebèkota in the Neoplatonic Commentaries on Plato and Aristotle », dans : T. Bénatouil, F. Trabattoni, G. Van Riel (éd.), Plato, Aristotle or Both? Dialogues between Platonism and Aristotelianism in Antiquity, Hildesheim.

Dillon, J.M. 1969 : «Plotinus, Enn. 3.9.1, and Later Views on the Intelligible World », Transactions and Proceedings of the American Philological Association, 100 (1969), p. 6370.

- 2003 : The Heirs of Plato: A Study of the Old Academy (347-274 BC), New York, 2003.

DoDDS, E.R. 1963: Proclus, The Elements fo Theology, a revised text with translation, introduction and commentary, Oxford, 21963.

ERLER, M. 1978 : Proklos Diadochos. Über die Existenz des Bösen, übers. und erl. von —, Meisenheim an Glan, 1978 (Beiträge zur klassichen Philologie, 102).

Ferrari, F. 2004 : Platone, Parmenide, introd. trad. e note, Milan, 2004 (Classici greci e latini).

FINE, G. 1993 : On Ideas. Aristotle's Criticism of Plato's Theory of Forms, New York, 1993.

FronterotTA, F. 2001 : ME $\Theta E \Xi I \Sigma$. La teoria platonica delle idee e la partecipazione delle cose empiriche, dai dialoghi giovanili al Parmenide, Pise, 2001 (Pubblicazioni della Classe di Lettere e Filosofia, Scuola Normale Superiore Pisa, 23).

Gerson, L.P. 1994 : Plotinus, Londres-New York, 1994 (The Arguments of the Philosophers).

GILL, M.L. 1996 : Plato, Parmenides, transl. by M.L. Gill \& P. Ryan, introd. by M.L. Gill, Indianapolis, 1996.

Kalligas, P. 1997 : «Forms of Individuals in Plotinus: A Re-Examination », Phronesis, 42 (1997), p. 206-227. 
LONGO, A. 2005 : Siriano e i principi della scienza, Naples, 2005 (Elenchos, 41).

Massagli, M. 1982 : «Amelio Neoplatonico e la metafisica del nous », Rivista di filosofia neoscolastica, 74 (1982), p. 225-243.

Miller, M.H. 1986 : Plato's Parmenides : The Conversion of the Soul, Princeton, 1986.

Morrow, G.R. \& J.M. Dillon 1987 : Proclus' Commentary on Plato's Parmenides, transl. by —, with introduction and notes by J.M. Dillon, Princeton, 1987.

Opsomer, J. 2003 : «La démiurgie des jeunes dieux selon Proclus », Les Études classiques, 71 (2003), p. 5-49.

- 2006: «Was sind irrationale Seelen?», dans: M. Perkams \& R.M. Piccione (éd.), Proklos. Methode, Seelenlebre, Metaphysik. Akten der Konferenz in Jena am 18.-20. September 2003, Leyde, 2006 (Philosophia antiqua, 98), p. 136-166.

Opsomer, J. \& C. STEEL 2003: Proclus. On the Existence of Evils, translated by Londres, 2003 (The Ancient Commentators on Aristotle, 50).

SAffrey, H.-D. \& L.G. WeSTERINK 1968 : Proclus. Théologie platonicienne, texte établi et traduit par —, vol. 1, Paris, 1968 (Collection des Universités de France).

SORABJI, R. 2004 : The Philosophy of the Commentators, 200-600 AD: 400 Years of Transition. A Sourcebook, vol. 3: Logic and Metaphysics, ed. by -, Londres, 2004.

Steel, C. 2007-2009 : Procli in Platonis Parmenidem commentaria ed. C. Steel, recognoverunt brevique adnotatione critica instruxerunt C. Steel, C. Macé, P. d'Hoine, 3 vol., Oxford, 2007-2009 (Oxford Classical Texts).

- 2008 : "Proclus on the Mirror as a Metaphor of Participation », dans : D. De Smet, M. Sebti et G. De Callataÿ (éd.), Miroir et savoir. La transmission d'un thème platonicien des Alexandrins à la philosophie arabo-musulmane, Leuven, 2008 (Ancient and Medieval Philosophy, De Wulf-Mansion Centre, I 38), p. 79-96.

Zoumpos, A.N. 1956 : Amelii Neoplatonici Fragmenta, collegit —-, Athènes, 1956. 